\title{
Active microcavity and coupled cavities in one-dimensional photonic crystal
}

\section{Biallo}

\section{A. D'Orazio}

dorazio@poliba.it

\section{Petruzzelli}

Dipartimento di Elettrotecnica ed Elettronica, Politecnico di Bari,Via Orabona 4, 70124 Bari, Italy

Dipartimento di Elettrotecnica ed Elettronica, Politecnico di Bari,Via Orabona 4, 70124 Bari, Italy

The propagation of light in one-dimensional $\mathrm{SiO}_{2}-\mathrm{TiO}_{2}$ coupled cavity photonic crystal is investigated. In particular the potential application in light amplification is proposed considering the small group velocity that characterizes the propagation at the edge of the resonance band due to the defects. Then, by means of a transfer-matrix method and a mode matching method code, an estimation of the photon lifetime and of the field intensity in a three-coupled cavity-photonic crystal is reported comparing it with those pertaining to a microcavity photonic crystal. This calculation allows us to underline the role of the light-matter interaction time with respect to that of the number of the active medium layers in the optical amplification. [DOI: 10.2971/jeos.2007.07010]

Keywords: photonic crystal, coupled-cavity, erbium

\section{INTRODUCTION}

The propagation of light through photonic band gap (PBG) structures [1] has been the focus of several investigations in the recent past. Their capability to control the flow of the light makes them very attractive for a lot of applications in numerous different fields like integrated optics, telecommunication and sensing. Since it was demonstrated that the photonic crystals (PCs) can slow down the propagating pulses, thanks to their unique dispersive properties $[2,3]$, the interest versus these structures is increased a lot. In fact slow light is so attractive for photonic devices because it leads to more efficient linear and nonlinear interaction [4]; in particular the long interaction time between radiation field and the matter caused by the small group velocity near the edge of the photonic band gap can enhance the gain of an active PC $[5,6]$. The stopping of the light can also be achieved by introducing a defect in the periodic lattice; this provokes a transmission resonance inside the band gap, that results in a high-Q field localization in the well bounded zone of the defect [7]. In this way, higher is the lifetime of the photon inside the microcavity region higher will be the efficiency of any non-linear interaction as self-phase modulation [4], second harmonic generation [8] or optical amplification [6]. Recently, it has been demonstrated guiding and bending of electromagnetic waves along a periodic arrangement of defects inside a three-dimensional photonic crystal at microwave frequencies [9]; in the coupled-cavity structures, photons hop from one evanescent defect mode to the neighbouring one due to overlapping between the tightly confined modes at each defect site [9]-[11]. Furthermore it has been numerically and experimentally shown that by placing more than one defect in cascade the light is guided through the coupled microcavity very slowly [12]. This phenomenon could be suitable to design an optical compact amplifier if an active medium is introduced in the structure.

In this paper we investigate the propagation of the slow modes in a coupled cavity (CC) realized in a one-dimensional $\mathrm{SiO}_{2}-\mathrm{TiO}_{2}$ photonic crystal [13]; in particular we estimate the photon lifetime and the field intensity in such a structure. By erbium doping the $\mathrm{SiO}_{2}$ layers we calculate the transmission coefficient focusing on a comparison between the performance of a CC structure and a photonic crystal microcavity (PCM). The comparison is made by considering two localised state resonances characterized by the same quality factor.

\section{NUMERICAL ANALYSIS}

The analysis of the CC structure and the PCM is performed by means of a transfer-matrix-method-based code [13, 14] while the simulations of the active CC and PCM are performed by considering a finite difference time domain (FDTD) approach [15]. The 1D PC investigated is a quarter-wave Bragg stack consisting of alternated layers of $\mathrm{SiO}_{2}$ and $\mathrm{TiO}_{2}$; the refractive indices of the silica and titania layers are $n_{s}=1.453$ and $n_{t}=2.304$ at the wavelength $\lambda=1.55 \mu \mathrm{m}$, respectively. The Bragg wavelength has been fixed to $\lambda_{B}=1.532 \mu \mathrm{m}$, where the erbium emission cross section is centered. The introduction of a defect half-wave long inside the periodic structure makes arise a resonance at the center of the band gap; then we consider a PCM constituted by 20 periods, 10 on the right and 10 on the left of the central $\mathrm{SiO}_{2}$ local defect (see Figure 1a). The localized state depicted in Figure $1 \mathrm{~b}$ is characterized by a cold-cavity quality factor $\mathrm{Q} \approx 15320$. 


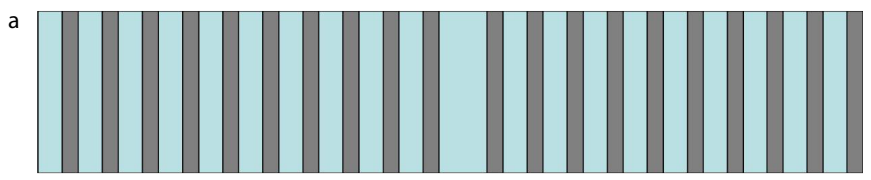

b

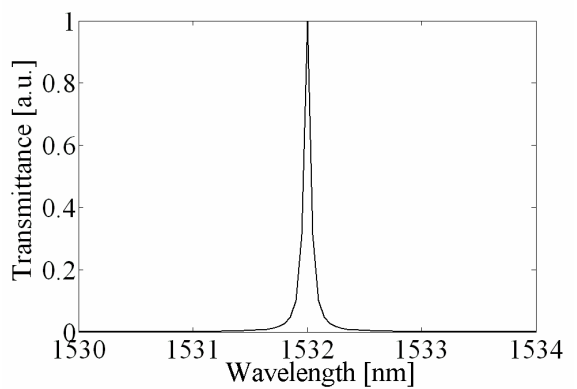

FIC. 1 a) 1D-PCM. b) Localized state resonance of the structure in Figure $1 a$; it is tuned at $\lambda_{B}=1.532 \mu \mathrm{m}$ and is characterized by a quality factor $Q \approx 15320$.

By adding another defect, two resonances appear at wavelengths shifted of the same quantity with respect to $\lambda_{B}=$ $1.532 \mu \mathrm{m}$. The two localized states move towards $\lambda_{B}$ as the separation between the defects increases and consequently the coupling between them decreases; Figure 2 shows that for a separation $\Lambda=10$ periods, we obtain a single frequency tuned at $\lambda_{B}$ characterized by a quality factor $Q \approx 510$, that is about six times smaller than that of the single cavity shown in Figure 1b.

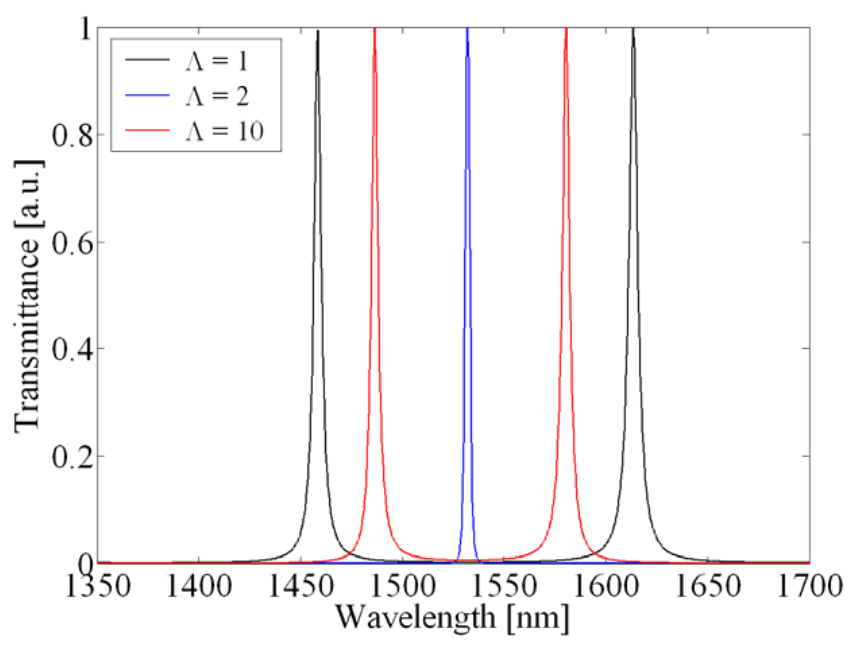

FIC. 2 Localized state resonances of a 2-coupled cavity-photonic crystal. By increasing the spacing between the two coupled cavities the two resonances move towards $\lambda_{B}$ until reaching one single resonance tuned at $\lambda_{B}$. The intercavity distance is $\Lambda$ and quoted per integer number of periods.

Thus, a third defect is introduced: Figure 3a depicts the designed 3-CC-PC structure in order to have a central resonance characterized by a quality factor $Q \approx 15320$ as for the PCM. In this case the frequency transmission spectrum of the structure shows three different resonances inside the bandgap, one of which is centered at $\lambda_{B}$ (see Figure $3 b$ ). By looking at Figure $3 c$, it is possible to observe that the two localized states match each other with a very high accuracy, allowing a rea- sonable comparison between the two investigated structures.
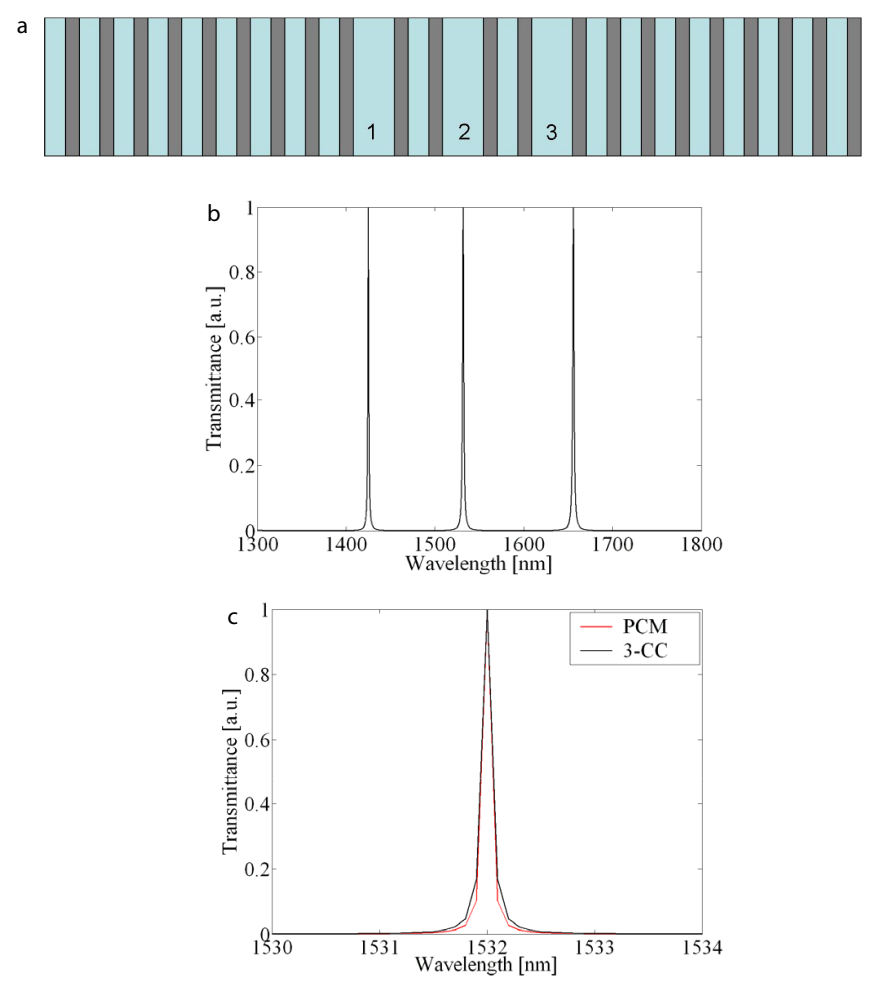

FIC. 3 a) 3-CC-photonic crystal with an intercavity distance of one period. b) Localized state resonance of the structure in Figure $3 \mathrm{a}$; the central resonance is tuned at $\lambda_{B}$. c) Comparison between the resonance in Figure $1 \mathrm{~b}$ and the central resonance in Figure 3b; the two localized states are charecterized by almost the same quality factor.

By considering a mode-matching technique the field intensity profile for a plane wave tuned at $\lambda_{B}$ is obtained and plotted in Figure $4 \mathrm{a}$ for the 3-CC-PC and in Figure $4 \mathrm{~b}$ for the single microcavity. While in the PCM the field is mainly concentrated in the defect, in the 3-CC-PC the field is localized overall in the lateral defect showing a node in the central cavity; moreover the intensity value reached by the field in the PCM is about two and half times the value reached in the 3-CC-PC; this means that although the localized states are characterized by the same quality factor, the light spends more time in the defect of the PCM with respect to the 3-CC-PC.

The photon lifetime of each defect in the 3-CC structure has been evaluated, by considering each one as a Fabry-Perot cavity; this allows us to foresee the amplification performance of such a structure compared to the single microcavity constituted by a defect half-wave long embedded in a 20 periods one-dimensional photonic crystal. The photon lifetime $\tau_{c}$ of a Fabry-Perot cavity can be calculated as follows:

$$
\tau_{c}=-\frac{n L_{c}}{c \ln \sqrt{R_{1} R_{2}}}
$$

where $L_{c}$ is the cavity length, $n$ is the cavity refractive index, $c$ is the speed of light in vacuum, $R_{1}$ and $R_{2}$ are the reflectivity of the two mirrors aside the defect. Figure 5 a depicts the evaluated photon lifetime of the three defects in the investigated 3-CC-PC and of the PCM. It is possible to note that, in correspondence of $\lambda_{B}$, the photon lifetimes of the lateral de- 

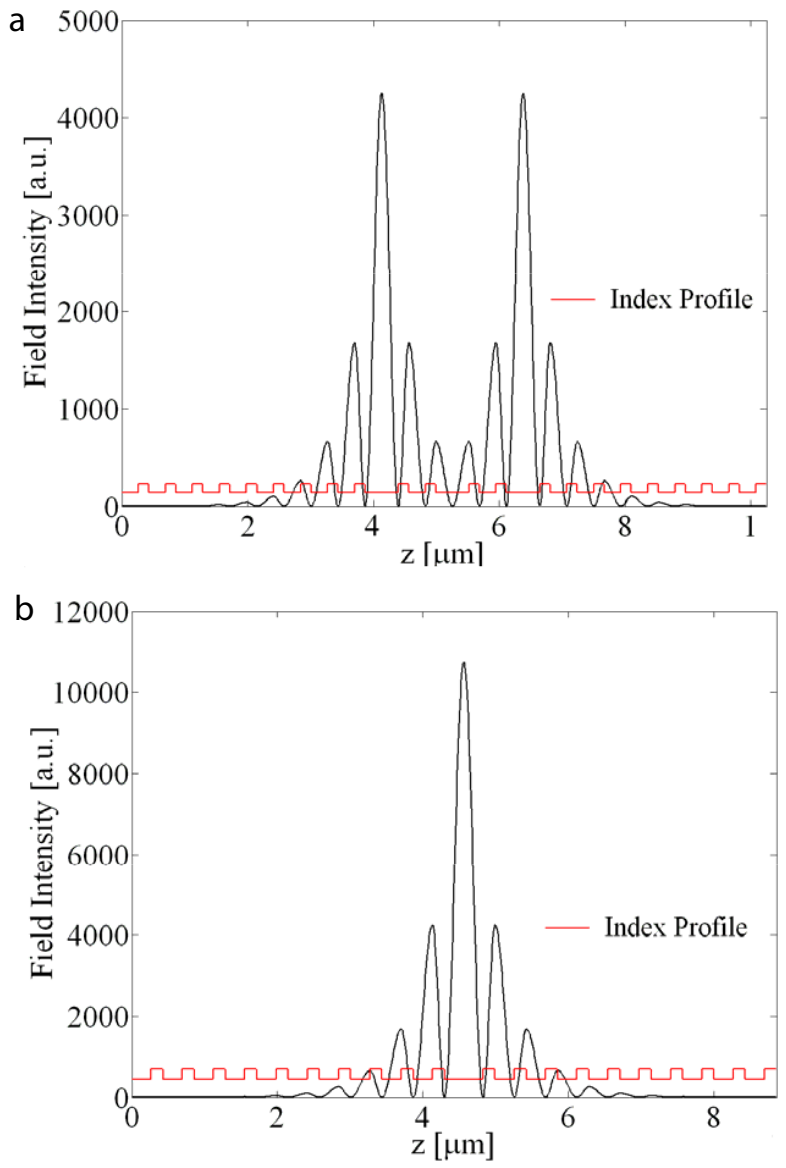

FIG. 4 a) Field intensity for a signal, tuned at $\lambda_{B}$, which through propagates through the 3 -CC. b) Field intensity for a signal, tuned at $\lambda_{B}$, which propagates through the PCM.

fects, $\tau_{1}$ and $\tau_{3}$ respectively, are bigger than that of the central defect $\tau_{2}$ verifying the results of the field intensity calculation; moreover the light stays in each of the three defects of the CC for a time that is more than three times smaller than that of the PCM. This results in a weaker interaction with the silica and so when the cavity is doped with erbium ions, the light interacts with the active medium more in the PCM than in 3CC-PC.

The analysis of the erbium doped CC amplifier has been performed by means of a home made computer code which implements a model [17] based on the auxiliary differential equation finite-difference time domain (ADE-FDTD) scheme [15] which solves together the Maxwell equations, the electron oscillator (EO) equation and the erbium rate equations that give the energy states population time variation during the propagation of pump and input signals. In particular the model considers the absorption and emission erbium cross sections by solving the EO equation (the ADE) that takes into account the field effect on the medium during its propagation [18]. For self-consistency, we briefly describe the implemented model referring the interested readers to the paper [17] for further details. The laser system is a four-level energy state one and the related rate equation model (Eqs. 2-6) considers the following energy state transitions: the ground state absorption (GSA), that occurs at the pump wavelength $\lambda_{p}=980 \mathrm{~nm}$, the stimulated emission (SE) that occurs at the input signal wave- length $\lambda_{a}=1532 \mathrm{~nm}$, where the peak of stimulated emission (SE) takes place, and the radiative and non-radiative transitions that are governed by the fluorescence times $\tau_{43}=\tau_{32}=$ $1 \cdot 10^{-9} \mathrm{~s}$ and $\tau_{21}=7.1 \cdot 10^{-3} \mathrm{~s}$. Moreover it includes the concentration quenching effects such as the up-conversion characterized by the coefficient rates $C_{3}=C_{u p}=5 \cdot 10^{-23} \mathrm{~m}^{3} / \mathrm{s}$ and the cross-relaxation characterized by the coefficient rate $C_{14}=3.5 \cdot 10^{-23} \mathrm{~m}^{3} / \mathrm{s}[19]$.

$$
\begin{gathered}
\frac{d N_{4}}{d t}=-\frac{N_{4}}{\tau_{43}}+C_{u p} N_{2}^{2}+C_{3} N_{3}^{2}-C_{14} N_{1} N_{4} \\
\frac{d N_{3}}{d t}=W_{p} N_{1}-\frac{N_{3}}{\tau_{32}}+\frac{N_{4}}{\tau_{43}}-2 C_{3} N_{3}^{2} \\
\frac{d N_{2}}{d t}=\frac{N_{3}}{\tau_{32}}+\frac{\mathbf{e}(t)}{h v_{s}} \frac{d \mathbf{p}(t)}{d t}-\frac{N_{2}}{\tau_{21}}+2 C_{14} N_{1} N_{4}-2 \cdot C_{u p} N_{2}^{2} \\
\frac{d N_{1}}{d t}=-W_{p} N_{1}-\frac{\mathbf{e}(t)}{h v_{s}} \frac{d \mathbf{p}(t)}{d t}+\frac{N_{2}}{\tau_{21}}-C_{14} N_{1} N_{4}+C_{u p} N_{2}^{2}+C_{3} N_{3}^{2}
\end{gathered}
$$

while the population density on different energy levels $\mathrm{N}_{i}$ are related to the total dopant concentration by the conservation equation:

$$
N_{T}=N_{1}+N_{2}+N_{3}+N_{4}
$$

The rate equations are linked to the propagating electric field $\mathbf{e}(\mathbf{t})$ and the macro-polarization vector $\mathbf{p}(t)$, by considering the EO equation:

$$
\frac{d^{2} \mathbf{p}(t)}{d t^{2}}+\Delta \omega_{a} \frac{d \mathbf{p}(t)}{d t}+\omega_{a}^{2} \mathbf{p}(t)=k \Delta N_{12}(t) \mathbf{e}(t)
$$

A Lorentzian susceptibility lineshape fits the experimentally measured erbium absorption and emission cross sections. The transmittance has been evaluated by calculating the FFT of the input and output electromagnetic field components and evaluating the ratio between the Poynting vector along the propagation direction at the output of the structure and the Poynting vector at the input of the structure.

For the FDTD simulations, a longitudinal step $\Delta z=5 \cdot 10^{-9} \mathrm{~m}$ and a time step $\Delta t=1.5 \cdot 10^{-17} \mathrm{~s}$, values which verify the Courant limit, have been considered. Each structure under analysis has been excited by a sinusoidal pump signal considering different power values and an input signal having a Gaussian lineshape centered around the erbium emission wavelength $\lambda=1.532 \mu \mathrm{m}$. The erbium ion concentration for both structures is $N_{t}=2.2 \cdot 10^{26}\left[\right.$ ions $\left./ \mathrm{m}^{3}\right]$ and the pump rate $W_{p}=11.42 \cdot 10^{4}$.

Figure $5 b$ depicts the transmission coefficient of the 3-CC-PC and of the PCM at $\lambda_{B}$; the PCM shows higher amplification with respect to the 3-CC-PC. This is not an obvious result because if it is true that the light stays into the PCM more time than in each of the three coupled cavity of the CC structure it is also true that in the 3-CC-PC there are more erbium doped layers. In an erbium doped waveguide amplifier longer is the structure higher is the gain so by increasing the number of erbium doped layers an increase of the transmission coefficient is expected. The 3-CC structure is longer than the PCM, having more doped defects; but, observing Figure 5b, one can see that the contribution to the gain due to the structure length isn't enough to fill up the gap in photon lifetimes between the 
two compared structures. It comes out that the light confinement in an active layer is more important than the total quantity of the active layers in a one-dimensional photonic crystal.
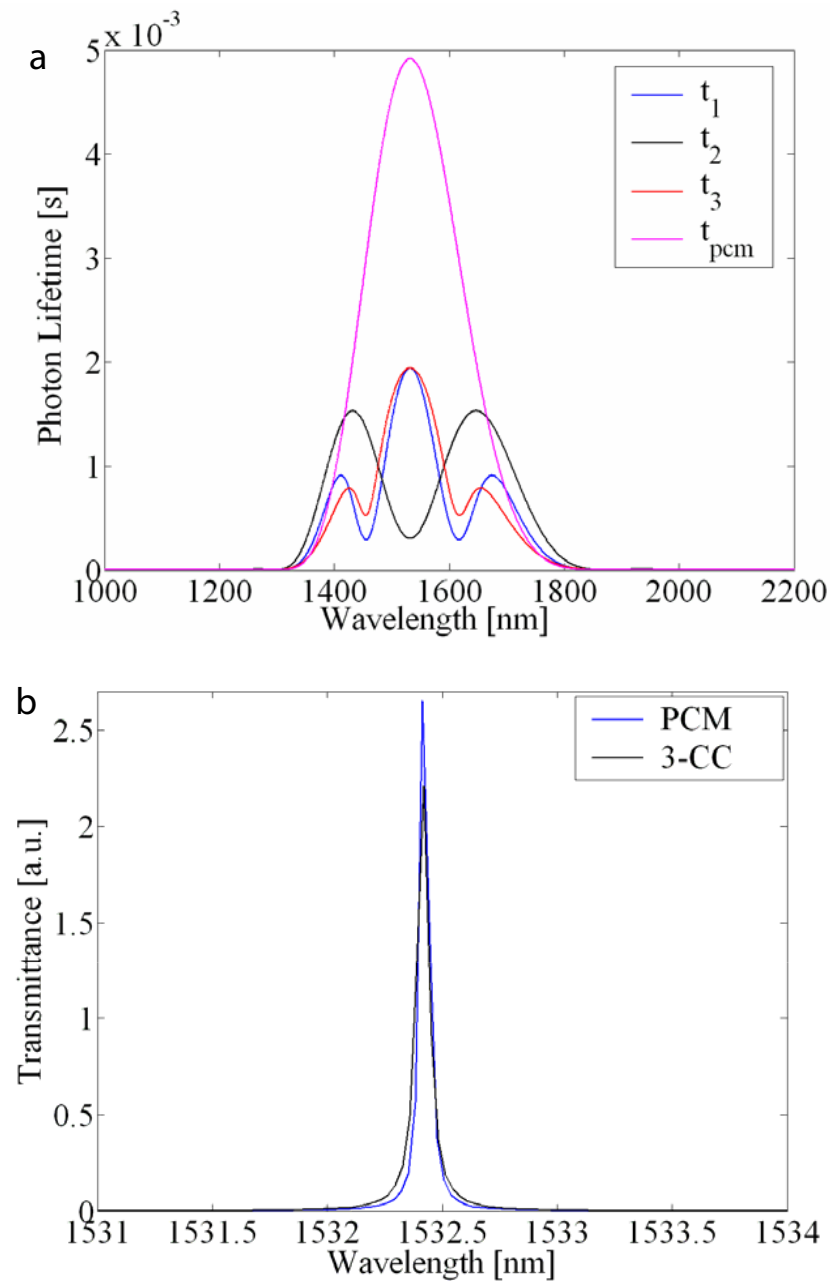

FIG. 5 a) Calculated photon lifetime of the $3-C C$ in Figure $3 a$ and of the PCM depicted in Figure $1 \mathrm{a}$, as a function of the wavelength. In particular, referring to the $3-\mathrm{CC}$ structure, $t_{1}$ and $t_{3}$ are the photon lifetimes of the lateral defects and $t_{2}$ the photon lifetime of the central defect. $t_{p c m}$ is the photon lifetime of the PCM. b) Transmission coefficient around $\lambda_{B}$ of the $3-C C-P C$ and the PCM doped with erbium.

\section{CONCLUSION}

This paper reports a comparison between a microcavity and 3coupled cavity in a one-dimensional $\mathrm{SiO}_{2}-\mathrm{TiO}_{2}$ photonic crystal for the amplification application. The estimation of the photon lifetime in each defect of both structures allows us to foresee the amplification performance of these devices when the silica layers are doped with erbium. In fact the optical amplification results to be dependent on the photon lifetime more strongly than on the number of active layers. For this reason photonic crystal microcavity configuration can be chosen to design the amplifier since it is more compact than the 3-coupled cavity amplifier to a parity of gain.

\section{References}

[1] J. D. Joannopoulos, R. D. Meade, J. N. Winn, Photonic Crystal: Molding the Flow of the Light (Princeton University Press, 1995).

[2] M. Centini, C. Sibilia, M. Scalora, G. D’Aguanno, M. Bertolotti, M. J. Bloemer, C. M. Bowden, I. Nefedov “Dispersive properties of finite, one-dimensional photonic band gap structures: Applications to nonlinear quadratic interactions" Phys. Rev. E 60, 4891-4898 (1999).

[3] J. P. Dowling, M. Scalora, M. Bloemer, C. M. Bowden, "The photonic band edge lasers: a new approach to gain enhancement" J. Appl. Phys. 75, 1896-1899 (1994).

[4] M. Soljacic, J. D. Joannopoulos, "Enhancement of nonlinear effects using photonic crystals" Nat. Mater. 3, 211-219 (2004).

[5] M. Botey, M. Maymò, D. Biallo, J. Martorell, "Photon lifetime at the inner band edges of a 3-D photonic crystal" Laser Phys. 14, 643-647 (2004).

[6] D. Biallo, A. D'0razio, V. Petruzzelli, "Enhanced light extraction in $\mathrm{Er}^{3+}$ doped $\mathrm{SiO}_{2}-\mathrm{TiO}_{2}$ microcavity embedded in one-dimensional photonic crystal" J. Non-Cryst. Solids 352, 3823-3828 (2006).

[7] D. J. Ripin, K. Lim, G. S. Petrich, P. R. Villeneuve, S. Fan, E. R. Thoen, J. D. Joannopoulos, E. P. Ippen, A. Kolodziejski, "One-dimensional photonic bandgap microcavities for strong optical confinement in GaAs and GaAs/Alx0y semiconductor waveguides" J. Lightwave Technol. 17, 2152-2160 (1999).

[8] M. Scalora, M. J. Bloemer, A. S. Manka, J. P. Dowling, C. M. Bowden, R. Viswanathan, J. W. Haus, "Pulsed second-harmonic generation in nonlinear, one-dimensional, periodic structures" Phys. Rev. A 56, 3166-3174 (1997).

[9] M. Bayindir, B. Temelkuran, E. Ozbay, "Tight-Binding Description of the Coupled Defect Modes in Three-Dimensional Photonic Crystals" Phys. Rev. Lett. 84, 2140-2143 (2000).

[10] M. Bayindir, S. Tanriseven, E. Ozbay, "Propagation of light through localized couplet-cavity modes in one-dimensional photonic bandgap structures" Appl. Phys. A 72, 117-119 (2001).

[11] M. Bayindir, C. Kural, E.Ozbay, "Coupled optical microcavities in one-dimensional photonic bandgap structures" J. Opt. A: Pure Appl. Op. 3, S184-S189 (2001).

[12] H. Altug, J. Vuckovic, "Experimental demonstration of the slow group velocity of light in twodimensional coupled photonic crystal microcavity arrays" http://arxiv.org/ftp/physics/papers/0406/0406109.pdf.

[13] A. Yariv, P. Yeh, Optical Waves in Crystals: Propagation and Control of Laser Radiation (John Wiley At Sons, 1984).

[14] J. M. Bendickson, J. P. Dowling, M. Scalora, "Analytic expressions for the electromagnetic mode density in finite, one-dimensional, photonic band-gap structures", Phys. Rev. E 53, 4107-4121 (1996).

[15] A. Taflove, S. C. Hagness, Computational Electrodynamics: the Finite-Difference Time-Domain Method (Artech House, BostonLondon, 2000).

[16] J. M. Liu, Photonic Devices (Cambridge University Press, 2005).

[17] D. Biallo, A. D’0razio, M. De Sario, V. Petruzzelli, F. Prudenzano, "Time domain analysis of optical amplification in $\mathrm{Er}^{3+}$ doped $\mathrm{SiO}_{2}$ $\mathrm{TiO}_{2}$ planar waveguide" Opt. Express 13, 4683-4692, (2005).

[18] A. E. Siegman, Lasers, (University Science Book, 1986).

[19] A. D’Orazio, M. De Sario, L. Mescia, V. Petruzzelli, F. Prudenzano, A. Chiasera, M. Montagna, C. Tosello, M. Ferrari, "Design of $\mathrm{Er}^{3+}$ Doped $\mathrm{SiO}_{2}-\mathrm{TiO}_{2}$ Planar Waveguide Amplifier," J. Non-Cryst. Solids $322,278-283$ (2003). 